\title{
Sobre el establecimiento de la sintonía en la primera sesión y la defensa del paciente
}

\section{About the stablishment of attunement during the first session and the patient's defense}

\author{
David maldavsky \\ Universidad de Ciencias Empresariales y Sociales, Argentina \\ (Rec.: junio de 2017 - Acept.: octubre de 2017)
}

\begin{abstract}
Resumen
Tras definir el concepto de sintonía, describir la muestra (la primera sesión o entrevista inicial de 20 pacientes con sus respectivos terapeutas) y el método (el algoritmo David Liberman, ADL), y presentar el repertorio de las intervenciones prototípicas del terapeuta, el trabajo analiza el establecimiento del vínculo de sintonía entre paciente y terapeuta y sus perturbaciones, así como las defensas del paciente durante la sesión en el momento de establecer la sintonía. Luego el trabajo procura establecer nexos entre los resultados referidos a las dificultades para lograr la sintonía y los resultados de los estudios de las defensas y su estado al intentar sintonizar con el paciente. El trabajo concluye que importa más el estado de la defensa que la severidad clínica que esta ponga de relieve.
\end{abstract}

Palabras clave: sintonía, defensas, intervenciones clínicas.

\begin{abstract}
After defining the concept of attunement, describing the sample (20 patients having gone through their first session or first interview with their corresponding therapists), defining the method (the David Liberman algorithm, DLA), and presenting the repertoire of the prototypical interventions of therapist, the paper analyzes the establishment of attunement between therapist and patient, the perturbations on the tuning, as well as the defenses used by patients while the attunement is established. Then the paper tries to establish a link between the results regarding difficulties on accomplishing attunement and the results from studying the patients' defenses and the state of defense when the therapist attempts to tune with the patient. The paper concludes that for establishing attunement, it is more relevant the state of the patients' defense rather than the clinical severity of this mechanism.
\end{abstract}

Keywords: attunement, defenses, clinical interventions.

\footnotetext{
* Correspondencia: David Maldavsky. Universidad de ciencias empresariales y sociales. Argentina.
} Email: dmaldavsky@gmail.com 


\section{Introducción}

Freud (1913) aseguró que el desarrollo de una actitud empática del terapeuta respecto al paciente es un requisito para la tarea clínica. Autores posteriores como Bolognini (2004), Eagle y Wolitzky (1997), Ferenczi (1928), Greenson (1965), Kohut (1959), Olden (1958), Pigman (1995), Schafer (1959), Trop y Storolow (1997) y Widlocher (2004), se enfocaron en la empatía, con similitudes y diferencias respecto de su definición, a su importancia clínica, sus componentes psíquicos y la relación con el paciente. Para fines de este trabajo, considero que la empatía involucra un cierto grado de identificación con el paciente que permite captar su estado afectivo. En cambio, la sintonía implica una armonía en la conexión entre dos o más personas, una conexión entre sistemas de emisión y recepción, y una igualdad de tono o frecuencia entre sistemas de vibraciones. En particular este último rasgo, ligado con la relación entre frecuencias, es afín con la descripción freudiana de las relaciones inter-neuronales y la circulación de la energía neuronal y pulsional, como fundamento de la conciencia originaria, la que puede hallarse perturbada en algunos pacientes con afecciones psicosomáticas, adicciones, epilepsias, neurosis post-traumática, etc. Prefiero denominar a las conexiones del terapeuta con ambos estados del paciente (el afectivo y el somático), como sintonía, más que como empatía, término que es preferible reservar para la conexión del terapeuta con los estados afectivos del paciente.

Existen pocos estudios sistemáticos sobre la sintonía con el paciente (Kantrowitz et al., 1989; Bohart \& Greenberg, 1997). En esta oportunidad me propongo estudiar la sintonía en el primer contacto, durante la primera sesión o primera entrevista, y su relación con las defensas y su estado en el paciente.

\section{Método}

\section{Participantes}

Recurriré a la transcripción de la primera sesión o entrevista de 20 pacientes con sus respectivos terapeutas: 1) Tupac, 2) Jacinta, 3) Estefanía, 4) Isabel, 5) Cátulo, 6) Carmen, 7) Favio, 8) Ms. Smithfield, 9) Ruby, 10) Celina, 11) Roberto, 12) Belisario, 13) Poncia, 14) Sonia, 15) Jimeno, 16) Serafín, 17) Corina, 18) Z, 19) Froilán, 20) Norberto. Cada sesión ha sido estudiada con anterioridad como caso único: a) en cuanto a las defensas (y su estado) tanto en las relaciones extra-transferenciales del paciente como durante la sesión, y b) en cuanto a las intervenciones de sus respectivos terapeutas, teniendo en cuenta su mayor o menor pertinencia clínica (Maldavsky, 2004, 2008; Maldavsky et al., 2005; Maldavsky et al., 2007a; Maldavsky et al., 2007b, Maldavsky et al., 2007c, Maldavsky et al., 2007d, Maldavsky et al., 2007e; Maldavsky et al., 2017).

La muestra está compuesta en un 50\% por la primera sesión de tratamiento, en un $45 \%$ por una entrevista inicial de admisión en una institución de psicoterapia, y en un $5 \%$ por una entrevista en un hospital psiquiátrico, en el marco de la "ronda de pacientes". En todos los casos se trata de los primeros contactos del terapeuta con los pacientes, quienes presentaban diferente grado de severidad, incluyendo un par con afasia leve. Los terapeutas, a su vez, tenían más de 10 años de práctica clínica con orientación psicoanalítica.
Instrumento para distinguir la sintonía del terapeuta con el paciente (primer paso)

Durante la primera sesión, las intervenciones del terapeuta parecen seguir una secuencia prototípica: 1) el terapeuta comienza realizando intervenciones que apuntan a establecer o mantener el contacto (saludos, frases de acompañamiento), seguidas de otras destinadas a obtener información acerca de eventos actuales o históricos de la vida del paciente, todas ellas con un valor "introductorio", y luego, 2) formula intervenciones ("centrales") de dos tipos, las que apuntan a focalizar el trabajo clínico y a sintonizar con el paciente, en particular con su estado afectivo y/u orgánico respecto de determinadas escenas narradas o desplegadas en sesión, y las que apuntan a proponer nexos, como relaciones causales, de semejanza, entre otras alternativas. Proponemos una taxonomía de las intervenciones tomando en cuenta su función, y en consecuencia distinguimos las intervenciones 1) introductorias (saludos, recabar información), 2) centrales (de sintonía y foco, y de establecimiento de nexos causales, de semejanza, etc.), y 3) complementarias (ejemplificaciones, síntesis, aclaraciones). En esta oportunidad solo nos concentraremos en las intervenciones introductorias y centrales, sobre todo las de sintonía y foco. Existen intervenciones no prototípicas que el terapeuta realiza ocasionalmente (completar frases interrumpidas del paciente, decirle que está ocultando información, etc.), que no forman parte de la secuencia más permanente.

Aunque esta secuencia no siempre sea pertinente desde la perspectiva clínica, pareciera ser la más usual. Esta secuencia presenta pequeñas variaciones (por ejemplo, el intento de sintonizar con el paciente puede estar compuesto por una o varias intervenciones, etc.). La tentativa de sintonizar con el paciente implica que el terapeuta ha elegido, entre la información recibida, un fragmento que considera importante para entender la situación presente. El paso siguiente, en el cual el terapeuta establece nexos, etc., solo puede desplegarse luego de alcanzada la sintonía y establecido el foco, debido a que la orientación clínica básica se origina en esta fase.

Es conveniente ofrecer un ejemplo de las intervenciones prototípicas y su secuencia. En la primera sesión de Jacinta (42 años), sonriendo, preguntó: “¿Por dónde empiezo?" Dijo que había estado pensando qué decir durante todo el día. Acto seguido, llamando al terapeuta por su nombre, le preguntó si podía tutearlo. El terapeuta aceptó la propuesta y Jacinta contó que había estado antes en terapia, pero no de manera regular. Agregó que necesitaba resolver ciertos problemas. Necesitaba entender qué le estaba pasando con los hombres, así como también sus problemas con sus padres y un nuevo proyecto de trabajo. No sabía cómo empezar, como si nunca hubiera estado en esa posición. La situación era extraña. El terapeuta -que hasta entonces había permanecido en silencio, excepto algunos sonidos (tales como "mhm")- le preguntó "por qué". La paciente respondió que recientemente había tenido algunos logros, pero que era conveniente. "No sé", agregó. El terapeuta resumió los tres problemas mencionados por Jacinta (trabajo, padres, hombres) y le preguntó por cuál prefería empezar. La paciente eligió un tema: su trabajo. Por varios años había trabajado como supervisora en una compañía del Estado y ahora tenía la posibilidad de obtener un cargo similar en una empresa privada. El terapeuta le preguntó si ambas posiciones eran recíprocamente excluyentes. La paciente dio respuestas imprecisas (descripción del tipo 
de trabajo, las relaciones jerárquicas internas, etc.), pero ante la insistencia del terapeuta, dijo que si tomaba el nuevo cargo debía renunciar al anterior. Temía que algo pudiera distraerla durante la evaluación para el nuevo trabajo. Ella se preguntaba por qué quería dejar el cargo actual. Allí se sentía tranquila, pero sabía que tenía que cambiar.

El terapeuta le preguntó si tenía miedo y Jacinta dijo que sí. Entonces el terapeuta formuló su primera interpretación: la paciente temía que su miedo fuera superior a sus deseos. La paciente asintió. Agregó que el trabajo actual quedaba a pocos metros de su casa. Dijo que era soltera y vivía en la casa de sus padres. Se había comprado un departamento para sí, pero lo vendió y sus padres se mudaron a otra casa. Ello no toleraba permanecer mucho tiempo en la casa en que vivían ahora sus padres. Allí no tenía su propio espacio. Su padre ocupó el cuarto que antes fue de ella. El terapeuta le interpretó que la cuestión del trabajo era el escenario donde ella desplegaba algo que tenía un común denominador con los otros temas: un conflicto entre por un lado lo nuevo, lo desconocido, conectado con la aventura y la ambición, y por el otro lado la seguridad, donde la sorpresa no tenía lugar. Relacionó este conflicto con el hecho de que su miedo pudiera ser superior a sus deseos. La paciente pasó entonces a comentar una situación laboral con sus compañeras en que apareció un conflicto entre el lucimiento y el sentirse víctima de maltrato. Como consecuencia de esta situación laboral terminó enferma.

El análisis de los relatos y de los actos del habla de la paciente condujo a resultados coincidentes: tanto en las relaciones extra-transferenciales como durante la sesión, predominaron defensas neuróticas fracasadas.

Durante la primera parte de la sesión las intervenciones del terapeuta se orientaron hacia el establecimiento del contacto con la paciente y la obtención de información. Luego el terapeuta se refirió a los estados afectivos de la paciente (intento de sintonía) respecto de su nuevo trabajo, y estableció nexos entre diferentes aspectos del material, elaboró generalizaciones e inferencias, etc.

\section{Instrumentos para el estudio de las defensas y su estado en el paciente (segundo paso)}

Las herramientas del algoritmo David Liberman (ADL) permiten detectar las defensas normales y patológicas (así como su estado) en las manifestaciones de paciente y terapeuta. El resultado de los estudios con el ADL sugiere la coexistencia de varias defensas centrales, entre las cuales una suele ser dominante. Con respecto al estado de la defensa, el ADL tiene sensibilidad para detectar tres alternativas: 1) exitoso, 2) fracasado, o 3) ambos (exitoso/fracasado). Cuando la defensa es exitosa se combinan a) el rechazo de determinados contenidos conflictivos, y b) sentimientos eufóricos. Cuando la defensa fracasa, a) lo rechazado retorna y b) predominan los sentimientos disfóricos. Cuando la defensa es exitoso-fracasada se combinan, a) el rechazo de algún contenido conflictivo, o a la inversa, el retorno de lo rechazado, y b) sentimientos disfóricos. La defensa exitoso-fracasada corresponde al repliegue psíquico (Maldavsky et al, 2005). De esta manera, por ejemplo, la desestimación del afecto tiene tres estados posibles: 1) exitoso: euforia química, 2) fracasado: angustia automática, 3) exitoso/fracasado: apatía.
El método consta de instrumentos para detectar las defensas en el nivel extra-transferencial (a través del análisis de los relatos del paciente) así como durante la sesión (mediante el análisis de los actos del habla, los componentes paraverbales y los movimientos del paciente). En ambos niveles de análisis (respectivamente, extra-transferencial e intra-sesión) el ADL detecta escenas (relatadas y desplegadas durante la sesión). También es posible comparar los resultados de ambos análisis (Maldavsky, 2013, Maldavsky et al., 2005; Maldavsky et al., 2007). Aún más, el ADL permite analizar la participación del terapeuta en esas escenas, en particular en las desplegadas en sesión.

El repertorio de defensas lleva a diferenciar entre: a) las funcionales, b) las de las neurosis de transferencia y caracterologías neuróticas, c) las de los rasgos de carácter narcisista no psicóticos, d) las de las psicosis, e) las de las adicciones, desórdenes psicosomáticos y neurosis traumáticas (Tabla 1). El repertorio propuesto es parte de una categorización freudiana de las estructuras clínicas, que es esencialmente dinámica y se centra en la relación entre conflictos y defensas. La noción de una defensa específica en las psicosis y en las patologías tóxicas y traumáticas, deriva del desarrollo de ideas de Freud (1918), Lacan (1957-58), McDougall (1978) y Maldavsky (1986, 1992). 
Tabla 1. Similitudes y diferencias entre las defensas principales

Similitudes y diferencias entre desmentida, desestimación, acorde a fines, creatividad y sublimación

\begin{tabular}{|c|c|c|c|c|}
\hline Defensa & Se opone a & Procedimiento & Recurso & Estructura clínica \\
\hline $\begin{array}{l}\text { Desmentida } \\
\text { (Verleugnung) }\end{array}$ & & $\begin{array}{l}\text { Refutación del juicio objetivo } \\
\text { y/o crítico }\end{array}$ & $\begin{array}{l}\text { Extraído de la realidad } \\
\text { objetiva (p.e. fetichismo) }\end{array}$ & $\begin{array}{c}\text { Rasgos patológicos } \\
\text { narcisistas de carácter } \\
\text { (esquizoides, depresivas, } \\
\text { paranoides, sobreadap- } \\
\text { tados) }\end{array}$ \\
\hline $\begin{array}{l}\text { Desestimación } \\
\text { (Verwerfung) }\end{array}$ & $\begin{array}{l}\text { Percepción y/o } \\
\text { afectos, juicios obje- } \\
\text { tivos, juicios críticos } \\
\text { contra el yo }\end{array}$ & $\begin{array}{l}\text { Abolición del yo que formula } \\
\text { el juicio objetivo y/o crítico } \\
\text { (yo real definitivo) o del yo } \\
\text { sujeto del afecto (yo real } \\
\text { primitivo) }\end{array}$ & $\begin{array}{c}\text { Producido en el yo mismo } \\
\text { como sustituto de la reali- } \\
\text { dad abolida (p.e. alucina- } \\
\text { ción o cuentas) }\end{array}$ & $\begin{array}{c}\text { Psicosis (esquizofrenia, } \\
\text { melancolía, paranoia) y } \\
\text { perturbaciones tóxicas y } \\
\text { traumáticas }\end{array}$ \\
\hline Acorde a fines & & $\begin{array}{l}\text { Transacción funcional entre } \\
\text { la pulsión (desexualizada), la } \\
\text { realidad y el superyó }\end{array}$ & $\begin{array}{l}\text { Actividad estudiantil o } \\
\text { laboral }\end{array}$ & --- \\
\hline Creatividad & & $\begin{array}{c}\text { Regresión formal del Pre- } \\
\text { consciente al funcionamiento } \\
\text { Inconsciente (con el aval del } \\
\text { superyó) }\end{array}$ & Humor, artes culinarias & --- \\
\hline Sublimación & & $\begin{array}{l}\text { Cambio de meta de la pul- } \\
\text { sión y elevación del ideal }\end{array}$ & Obra con un valor cultural & --- \\
\hline
\end{tabular}

Similitudes y diferencias entre represión, acorde a fines, creatividad y sublimación

\begin{tabular}{|c|c|c|c|c|}
\hline Defensa & Se opone a & Procedimiento & Recurso & Estructura clínica \\
\hline Represión & & $\begin{array}{l}\text { Desinvestidura Precons- } \\
\text { ciente (que no da cabida a } \\
\text { contenidos Inconscientes) y } \\
\text { contrainvestidura Precons- } \\
\text { ciente }\end{array}$ & Formación sustitutiva & $\begin{array}{l}\text { Neurosis de } \\
\text { transferencia }\end{array}$ \\
\hline Acorde a fines & Deseos & $\begin{array}{c}\text { Transacción funcional entre } \\
\text { la pulsión (desexualizada), la } \\
\text { realidad y el superyó }\end{array}$ & $\begin{array}{c}\text { Actividad estudiantil o } \\
\text { laboral }\end{array}$ & --- \\
\hline Creatividad & & $\begin{array}{c}\text { Regresión formal del Pre- } \\
\text { consciente al funcionamiento } \\
\text { Inconsciente (con el aval del } \\
\text { superyó) }\end{array}$ & Chiste & --- \\
\hline Sublimación & & $\begin{array}{c}\text { Cambio de meta de la } \\
\text { pulsión y elevación del ideal }\end{array}$ & Obra con un valor cultural & --- \\
\hline
\end{tabular}


Los instrumentos del ADL permiten estudiar los deseos y las defensas y su estado en las escenas relatadas y en las desplegadas por el paciente en la sesión.

\section{Procedimientos}

Los procedimientos siguen dos pasos. En el primero se realizan estudios sobre la tentativa de sintonía del terapeuta con el paciente en un conjunto de sesiones, y en el segundo, se analizan los deseos y las defensas en el paciente en el momento de establecer la sintonía. Este procedimiento implica que los instrumentos se aplican de manera sucesiva, primero el descrito en "Instrumento para distinguir la sintonía del terapeuta con el paciente (primer paso)" y luego los descritos en "Instrumentos para el estudio de las defensas y su estado en el paciente (segundo paso), en particular, el centrado en los deseos y defensas y su estado en los actos de habla del paciente. El diseño en dos pasos se asemeja a uno realizado con anterioridad (Maldavsky, Argibay, Scilletta, Rembado et al., 2016): en aquella oportunidad, en el primero el estudio clasificó las intervenciones del terapeuta y en el segundo, infirió los deseos de dicho terapeuta en los diferentes tipos de intervenciones. Aquel estudio tuvo un carácter cuali-cuantitativo, mientras que el actual es más claramente cualitativo.

\section{Análisis}

\section{Primer paso: La sintonía y sus interferencias en 11 casos}

\section{Resultados del estudio de cada caso}

Sintetizaré el análisis de las dificultades para establecer la sintonía en 11 de los casos estudiados en la secuencia de intervenciones clínicas. Las categorías para la evaluación son exitosa (cuando el terapeuta alcanzó su objetivo), fracasada (cuando no lo logró), incompleta (cuando lo logró solo parcialmente) y retrasada (cuando ocurre más tarde de lo usual, es decir, luego del segundo tercio). En esta síntesis interesa especialmente, describir las secuencias iniciales en el intercambio entre paciente y terapeuta, y que corresponden, en el terapeuta, al pasaje desde las intervenciones introductorias hasta las intervenciones centrales (de sintonía y foco, y luego a las de nexo), y en el paciente, a las respuestas ante dichas intervenciones clínicas. Además de estos 11 casos (así como el de Jacinta, antes mencionado), existen estudios de la primera sesión de otros ocho pacientes: Tupac, Estefanía, Cátulo, Carmen, Favio, Ms. Smithfield, Ruby. En los casos de Tupac, Estefanía y Cátulo el terapeuta no tuvo dificultades en sintonizar con el paciente; lo mismo ocurrió con Jacinta. En los otros casos, las dificultades del terapeuta se asemejaron a las de otros participantes de este estudio.

Debido a la necesidad de sintetizar la descripción de cada problema, solo he indicado sus rasgos centrales, y transformé además cada caso en un ejemplo que puede generalizarse.

Carmen: a veces el intento del terapeuta de sintonizar con el paciente demora desproporcionadamente, ya que aquel necesita mucho tiempo para establecer el contacto inicial y obtener información de parte de este. En el período introductorio (de comienzo de la sesión), generalmente el terapeuta obtiene información que le permite orientarse para formular su primera intervención central, a la búsqueda de la sintonía con el paciente. Pero a veces la información que obtiene más bien resulta una interferencia, sea porque el terapeuta no queda convencido de lo que el paciente dice, sea porque este es reticente. En estas ocasiones el terapeuta se ve llevado a describir la situación que le impide pasar desde estas intervenciones introductorias hacia otras, centrales. El terapeuta combina entonces dos intervenciones: primero señala la actitud resistencial del paciente y luego agrega que si este mantiene dicha posición, él no podrá ayudarlo.

Celina: a veces la intervención elegida por el terapeuta para sintonizar con el paciente, puede no ser pertinente desde la perspectiva clínica. Así puede ocurrir cuando el paciente enfatiza excesivamente su estado afectivo en sesión, y el hecho de que el terapeuta haga también referencia a los sentimientos del paciente conduce a que este refuerce su postura.

Roberto: algunas veces sucede que las intervenciones dirigidas a sintonizar con el paciente no siguen la secuencia usual descrita poco antes. Dichas intervenciones quedan pospuestas y son reemplazadas por otros tipos de intervenciones (sobre todo aquellas destinadas a establecer relaciones causales), con lo cual el terapeuta corre el riesgo de seguir una orientación clínicamente no pertinente.

Belisario: ocasionalmente, mientras el terapeuta intenta sintonizar con el paciente, sabotea inadvertidamente su propia labor al incluir determinadas palabras o giros que podrían ser entendidos como indicadores de su falta de sensibilidad respecto de algún rasgo del consultante. En consecuencia, el paciente tiene dos alternativas: puede responder al intento del terapeuta de sintonizar con él, o puede responder a las palabras o giros del terapeuta que hacen de interferencia.

Poncia: en ocasiones el problema aparece al principio, en el momento en que el terapeuta establece el contacto y pretende obtener información, debido a que en dicha fase el terapeuta formula una intervención prematura, que muestra que puede notar rápidamente algún problema del paciente. Entonces, en un momento posterior, al formular intervenciones que apuntan a establecer nexos, realizar generalizaciones, etc., puede encontrarse con un rechazo sistemático del paciente. El problema aparece pues en la primera y en la tercera fase de la secuencia de intervenciones, y no en la segunda (sintonía).

Sonia: a veces el terapeuta tiene dos alternativas (en cuanto a estados) para establecer la sintonía: con sentimientos depresivos y con la angustia del paciente, y si opta por la primera alternativa, el proceso de sintonía queda incompleto, quedando también incompletas la focalización y la alusión a los nexos.

Isabel: a veces el terapeuta puede intentar sintonizar con el paciente pero, en lugar de poner el énfasis en el presente de la sesión, presta atención a las relaciones extra-transferenciales, que son secundarias.

Serafín: la sintonía del terapeuta con el paciente puede no necesariamente significar que aquel enfatice los sentimientos de este, sino su condición somática. En ocasiones, el terapeuta no advierte desde el comienzo que no es correcto adscribirle un estado afectivo a un paciente, dificultad que se ve incrementada porque además, ante una intervención del terapeuta que alude a un afecto del paciente, este puede corroborar que efectivamente lo siente. Entonces, el terapeuta puede permanecer desorientado por un tiempo, hasta que logra rectificar- 
se y pasa a enfatizar los estados somáticos del paciente, más que los psíquicos.

Z: a veces el establecimiento del contacto inicial con el paciente se ve obstaculizado cuando este sufre un estado general de desvitalización y apatía, y el terapeuta realiza esfuerzos por darle algo de vitalidad. De este modo, crea condiciones mínimas para el establecimiento de una relación terapéutica, lo cual puede interferir en el subsecuente intento de sintonía. Además, en lugar de concentrarse en la situación presente, el terapeuta puede tender a enfatizar el pasado del paciente, 0 puede incluso prestar atención a estados afectivos secundarios, y no a los centrales.

Froilán: en ocasiones los intentos del terapeuta de sintonizar con el paciente son obstaculizados por las observaciones y relatos de este último, que distraen la atención del tema central.

Norberto: a veces, ante un discurso resistencial del paciente, el terapeuta no atina a realizar intervenciones para establecer la sintonía durante la sesión, y en consecuencia permanece clínicamente desorientado.

\section{Dos descripciones más extensas}

Presentaremos ahora dos descripciones más extensas correspondientes al momento de la sintonía. Ellas representan finales opuestos del espectro: el caso de Carmen muestra problemas relativamente menos severos, mientras que el caso de Norberto presenta dificultades más importantes en el proceso de sintonía.

Carmen: La paciente (26 años) comenzó la primera sesión diciendo que consultaba porque había decidido ir a vivir sola, separada de los padres, y que entonces el novio se había mudado a su vivienda. El terapeuta le preguntó si el hecho de irse a vivir juntos había sido decidido entre ambos, a lo que Carmen respondió que había sido una decisión unilateral del novio. No obstante, enfatizó inmediatamente que lo importante era que esta situación le traería problemas con sus padres. Temía que sus padres se enteraran de su relación con él y se enojaran con ella. Aclaró que sus padres no gustaban de su novio y que ella había mantenido la relación entre ambos a escondidas durante años. Ahora le resultaba imposible continuar ocultando el vínculo, y no sabía qué hacer. La terapeuta exploró extensa y cuidadosamente el problema de la hostilidad de los padres de Carmen. Le preguntó a la paciente si podía dar algún ejemplo del enojo de los padres hacia ella, sin que Carmen pudiera aportar ninguno. Como pese a ello la paciente continuaba insistiendo en que ese era el problema central, la terapeuta le dijo que la consultante omitía información y que de esa manera no podría ayudarla. La paciente estuvo de acuerdo y la terapeuta volvió a la situación que había originado el problema: tomando una decisión unilateral, su novio se había mudado a la vivienda de la paciente. Esta dijo entonces que su novio había sufrido una adicción a la cocaína y que se había recuperado luego de haber tenido varias internaciones de rehabilitación. Entonces la terapeuta le preguntó si esta situación la angustiaba, y la paciente, tras responder afirmativamente, comenzó a concentrar su atención en su relación con el novio y disminuyó su resistencia.

En el momento inicial de la sesión, cuando la paciente omitía información, podía repetirse en el vínculo con la terapeuta la relación que aquella describía con los padres. Esta relación combinaba el rasgo caracterológico evitativo y una actitud desafiante secreta. En sus relaciones extra-transferenciales esta combinación de mecanismos había fracasado, de donde derivaba la angustia con la que consultó. Durante la primera parte de la sesión la paciente había intentado recuperar ante la terapeuta el carácter exitoso de estas defensas, pero luego se tornó más colaboradora y disminuyeron los rasgos evitativos y desafiantes. El tratamiento duró 5 años.

Norberto: Casi al comienzo de la primera sesión, el paciente (38 años) hizo una confesión: tenía un puesto importante en un cargo público pero carecía de un título que lo habilitara para ello. Para desembarazarse de la tensión que le causaba esta situación (a lo que se agregaba que, para tomar decisiones, siempre seguía sus propias opiniones en vez de basarse en los consejos de profesionales) recurría a fugaces encuentros homosexuales con hombres que conocía ocasionalmente en la calle. A ello se agregaba un nuevo problema: no lograba concretar un vínculo sexual con su esposa, pese a que ella se lo solicitaba discretamente. El terapeuta dedicó casi toda la hora a recabar información y, hacia el final, intervino con cautela: dijo que creía que el paciente era agresivo. El paciente no manifestó sorpresa al escuchar esta intervención, e incluso agregó algunos detalles que armonizaban con la opinión del terapeuta. Luego de finalizada la hora, el terapeuta temió haber sido hostil con el paciente. Poco después, mientras revisaba el material con mayor detenimiento, llegó a la conclusión de que había sido ingenuo. La defensa central del paciente, tanto en sus relaciones extra-transferenciales como durante la sesión, fue la desmentida exitosa combinada con la desestimación del afecto también exitosa. El paciente no volvió a concurrir al tratamiento.

Del total de la muestra nos hemos centrado en solo 11 casos. El resto de ellos no presentaron un problema significativo en relación con la sintonía (cuatro casos) o bien manifestaron las mismas dificultades que algunos de los otros (cinco casos).

Las dificultades en establecer la sintonía pueden agruparse en dos categorías: la primera con problemas transitorios y la segunda con problemas duraderos y en ocasiones, irresueltos. En el primer grupo aparecen estos problemas: 1) la resistencia de un paciente que intentó reinstalar, transitoriamente durante la sesión, los mecanismos evitativos que le habían fracasado en las relaciones extra-transferenciales, 2) el carácter no pertinente de una única intervención clínica que enfatizaba el sentir con un paciente que tendía a jerarquizar los afectos como resistencia, 3) el carácter prematuro de ciertas intervenciones centrales del terapeuta correspondientes a nexos causales, antes de intentar establecer la sintonía, 4) el autosabotaje transitorio del terapeuta al emplear, en la tentativa de sintonizar con el paciente, términos que este había explicitado que rechazaba, 5) la selección, entre dos alternativas pertinentes en cuanto al afecto (cada una de ellas acompañada de un diferente foco y de una estrategia consiguiente de intervenciones de nexo), solo de una de ellas, con las consecuencias en cuanto a la focalización y las intervenciones de nexo, 6) las dificultades para avanzar desde las intervenciones centrales (destinadas a sintonizar) hasta las intervenciones centrales destinadas a establecer nexos causales, etc., y 7) la realización de intervenciones no pertinentes para sintonizar con el paciente, hasta que el terapeuta se rectificó y logró su objetivo. Las primeras cuatro dificultades fueron solo transitorias. La 
dificultad mencionada en quinto lugar condujo a una resolución solo parcialmente satisfactoria. El problema mencionado en sexto lugar fue recurrente, mientras que el séptimo involucró la estrategia íntegra que el terapeuta adoptó al comienzo, que resultó errónea, y que luego pudo remplazar por otra, más pertinente, que le permitió sintonizar con el paciente.

En el segundo grupo encontramos dificultades más severas para establecer la sintonía. Estas son: 1) la atribución de determinado estado afectivo al paciente, en lugar de un estado somático, intervención que hubiera sido pertinente, 2) el énfasis en los estados afectivos desarrollados en las relaciones extra-transferenciales actuales en lugar de los que se daban durante la sesión, 3) el énfasis en los estados afectivos en el pasado del paciente, en lugar de los que se daban en su presente, 4) la jerarquización de determinado estado afectivo en el paciente, que no parecía ser el central, 5) la imposibilidad de superar la oposición del paciente cuando el terapeuta intentaba sintonizar con él, 6) la inexistencia de una tentativa de sintonía con el paciente. Los problemas 3 y 4 corresponden al mismo caso, $Z$.

Las dificultades más importantes del terapeuta para establecer la sintonía con el paciente ocurrieron en los casos de Isabel, Jimeno, Serafín, Corina, Froilán, Norberto y Z. En los casos de Jimeno, Corina, Froilán y Norberto, las dificultades podían atribuirse en gran medida a la resistencia de los pacientes, en cambio, en los casos de Serafín y de Z, la dificultad podía atribuirse a la manera en que el terapeuta conducía la entrevista, ante un paciente carente de energía. Estos 6 casos no continuaron el tratamiento o bien lo abandonaron luego de 2 o 3 sesiones.

Consideremos ahora aquellas situaciones (en que en que el terapeuta rectificó o no sus propias intervenciones y la eficacia clínica de su actitud. La terapeuta de Carmen interrumpió transitoriamente sus preguntas destinadas a obtener información y pasó a decirle que creía que estaba omitiendo parte de ella. Luego de ello pudo continuar con la tarea que había interrumpido. El terapeuta de Celina remplazó rápidamente su intento de sintonizar con la paciente, por el intento de establecer nexos, hacer generalizaciones, etc. El entrevistador de Roberto no continuó con su intento prematuro de establecer nexos causales, y pasó a intentar la sintonía con el paciente. El terapeuta de Belisario pudo dejar de emplear palabras que podían despertar el rechazo del paciente y las sustituyó por otras más adecuadas para establecer la sintonía clínica. El terapeuta de Poncia no rectificó su orientación clínica, por lo cual la sesión osciló entre momentos en que la paciente colaboraba con la tarea y ambos podían trabajar juntos de manera fructífera, y momentos de resistencia, sobre todo cuando el terapeuta intentaba establecer nexos. El terapeuta de Isabel pudo rectificar su orientación clínica y logró entonces establecer la sintonía. El terapeuta de Jimeno cambió su orientación clínica al aceptar la descripción del paciente de sus propios estados y, en consecuencia, pudo sintonizar con él y desarrollar una estrategia más pertinente. El terapeuta de Serafín logró rectificar la orientación para sintonizar con el paciente, pero no pudo dar el siguiente paso. La terapeuta de Corina no pudo acceder al pasaje desde recabar información hasta sintonizar con la paciente en el presente de la sesión. El terapeuta de $\mathrm{Z}$ no advirtió hasta luego de terminada la hora, el peso que tenía para el paciente la influencia materna, por lo cual no pudo obtener mayor información al respecto y sintoni- zar con el paciente. El terapeuta de Froilán y el de Norberto no realizaron intervenciones clínicas pertinentes.

En síntesis, cuatro de los terapeutas (los de Poncia, Z, Froilán y Norberto) no rectificaron su orientación clínica no pertinente, mientras que los restantes sí lo hicieron. No obstante, el terapeuta de Poncia no necesitó rectificar sus intervenciones relacionadas con la sintonía con la paciente, sino las ligadas con el establecimiento de nexos causales, generalizaciones, etc., que despertaban la permanente oposición de Poncia. A su vez, el terapeuta de Sonia no terminó de formular una sintonía completa y por lo tanto también quedó incompleta la focalización y el establecimiento de nexos.

Por otra parte, en otros seis casos (Carmen, Celina, Roberto, Belisario, Isabel y Jimeno) la rectificación del terapeuta condujo al desarrollo de una nueva estrategia completa (1. contacto y obtención de información, 2. sintonía, 3. establecimiento de nexos), mientras que con Corina y Serafín la rectificación del terapeuta tuvo un carácter parcial, y no fue posible desplegar una nueva estrategia completa.

La Tabla 2 sintetiza los intentos exitosos o fracasados del terapeuta de sintonizar con el paciente, en algunos casos incluyendo una segunda estrategia clínica.

En el caso 1 no hubo cambios en la defensa exitosa acorde a fines. En los casos 4, 17, 18, 19 y 20 no hubo cambios en las defensas patológicas. En los casos 2, 3, 5, 7 y 13 el cambio en las defensas ocurrió durante la recolección de la información. En el caso 16, el cambio se dio luego de un extenso período en que fracasó la tentativa de sintonía, y hacia el final de la hora el paciente cambió la defensa en un nuevo período de recolección de la información. En los casos 6, 8, 9, 10, 11, 12, 14 y 15 el cambio en la defensa se dio durante el momento de la sintonía. En el caso 9 la tentativa del terapeuta se dio mientras en la paciente predominaban defensas patológicas fracasadas, que en ese momento incrementaron su severidad.

En este análisis solo se estudia la defensa central y su estado en el momento de la sintonía. Algunos terapeutas (casos 1-5) pudieron sintonizar con sus pacientes fácilmente. Otros terapeutas (6-13) tuvieron dificultades transitorias con la sintonía, pero finalmente pudieron superarlas. Por último, un tercer grupo de terapeutas (casos 14-20) tuvo dificultades mayores. Los terapeutas 14, 15 y 16 finalmente pudieron sintonizar con sus pacientes. En cambio, los cuatro terapeutas restantes no lograron hacerlo. Con respecto a los pacientes, en los casos 17-20 el estado de la defensa central (exitoso-fracasado o exitoso) se mantuvo inalterado durante toda la sesión. Recordemos que cuando la defensa patológica central es exitosa, el paciente puede rechazar determinados contenidos conflictivos y mantener un estado eufórico, mientras que cuando la defensa es exitoso-fracasada, el estado del paciente usualmente se vuelve disfórico, pero no emerge la angustia, que exige mayor trabajo. Cuando la defensa patológica es exitosa, la resistencia es mayor, y disminuye un tanto cuando la defensa es exitoso-fracasada, aunque la colaboración del paciente en la tarea terapéutica es escasa. Este hecho podría explicar por qué la sintonía se halla dificultada. 
Tabla 2. Carácter exitoso o fracasado de la tentativa de sintonía del terapeuta con el paciente

\section{Segunda intervención central}

\section{Paciente}

1. Tupac

2. Jacinta

3. Estefania

4. Cátulo

5. Carmen

6. Favio

7. Ms. Smithfield

8. Ruby

9. Celina

10. Roberto

11. Belisario

12. Poncia

\section{Intervención introductoria}

Exitosa
Exitosa
Exitosa
Exitosa
Exitosa
Exitosa
Exitosa
Exitosa
Exitosa
Exitosa

Exitosa

Interferida parcialmente

\section{Primera intervención central}

Exitosa

Exitosa

Exitosa

Exitosa

Sintonía retrasada

Sintonía retrasada

Sintonía retrasada

Sintonía retrasada

Sintonía clínicamente

$$
\text { no-pertinente }
$$

Inversión de la secuencia de intervenciones (entre la tentativa de sintonizar y la de establecer nexos)

Auto-sabotaje

Exitosa
Exitosa

Exitosa

Exitosa

Exitosa

Exitosa

Exitosa

Exitosa

Exitosa

Exitosa

Exitosa

\section{Exitosa}

Interferencias en la secuencia de intervenciones (de la primera a la segunda intervención central)

$\begin{array}{llll}\text { 13. Sonia } & \text { Exitosa } & \text { Incompleta } & \text { Incompleta } \\ \text { 14. Isabel } & \text { Exitosa } & \text { Fracasada } & \text { Fracasada } \\ \text { 15. Jimeno } & \text { Exitosa } & \text { Fracasada } & \text { Fracasada } \\ \text { 16. Serafin } & \text { Fracasada } & \text { Fracasada } & \text { Fracasada } \\ \text { 17. Corina } & \text { Fracasada } & \text { Fracasada } & \text { Fracasada } \\ \text { 18. Z } & \text { Fracasada } & \text { Fracasada } & \text { Fracasada } \\ \text { 19. Froilán } & \text { Fracasada } & \text { Fracasada } & \text { Fracasada } \\ \text { 20. Norberto } & \text { Fracasada } & \text { Fracasada } & \text { Fracasada }\end{array}$

Segunda intervención clínica

\begin{tabular}{lccc}
\hline 14. Isabel & Exitosa & Exitosa & Exitosa \\
15. Jimeno & Exitosa & Exitosa & Exitosa \\
16. Serafín & Exitosa & Exitosa & Incompleta \\
17. Corina & Exitosa & Incompleta & Incompleta \\
18. Z & Exitosa & Incompleta & Incompleta \\
19. Froilán & Fracasada & Fracasada & Fracasada \\
20. Norberto & Fracasada & Fracasada & Fracasada \\
\hline
\end{tabular}




\section{Segundo paso: Modificación o persistencia del tipo y estado de la defensa en el momento en que el terapeuta intenta estable- cer la sintonía con el paciente}

Resultados del estudio de cada uno de los 20 casos

La Tabla 3 muestra panorámicamente el tipo y estado de la defensa y sus cambios en el paciente en el momento en que el terapeuta intenta establecer la sintonía.

Tabla 3. Modificación o persistencia del tipo y estado de la defensa en el momento de la sintonía

\section{Paciente Modificación o persistencia de la defensa y su estado}

\begin{tabular}{ll}
\hline $\begin{array}{l}\text { 1. Tupac } \\
\text { 2. Jacinta }\end{array}$ & $\begin{array}{l}\text { Defensa acorde a fines exitosa. } \\
\text { Pasaje desde los rasgos caracterológicos evitativos fracasados hasta un mecanismo de defensa acorde } \\
\text { a fines exitoso. }\end{array}$ \\
3. Estefa & Pasaje desde la desmentida fracasada hasta la represión fracasada. \\
4. Cátulo & Desestimación del afecto y desmentida, ambas fracasadas. \\
5.Carmen & Pasaje desde los rasgos caracterológicos evitativos exitosa a la defensa acorde a fines exitosa.
\end{tabular}

6. Favio Pasaje de la desmentida combinada con rasgos histriónicos de carácter desde un estado exitoso para ambos hasta uno fracasado para ambos.

7. Ms Smithfield Pasaje desde la desestimación del afecto exitosa hasta la defensa acorde a fines exitosa.

8. Ruby

9. Celina

10. Roberto

11. Belisario

12. Poncia

13. Sonia

14. Isabel

15. Jimeno

16.Serafín

17.Corina

18. Z

19. Froilán

20. Norberto
Pasaje desde la represión con rasgos caracterológicos histriónicos exitosa a la defensa acorde a fines exitosa

Pasaje desde la desestimación de la realidad y la instancia paterna fracasada hasta una combinación entre la desestimación del afecto y la desestimación de la realidad y la instancia paterna, ambas fracasadas.

Pasaje desde la desestimación del afecto exitosa hasta la represión exitosa.

Pasaje desde la desestimación del afecto fracasada hasta la desmentida fracasada.

Pasaje desde la desestimación del afecto exitosa hasta la defensa acorde a fines exitosa.

Pasaje desde la desestimación del afecto exitosa hasta la defensa acorde a fines exitosa.

Pasaje de la desmentida desde el estado exitoso hasta el fracasado.

Pasaje desde la desestimación del afecto y la desmentida fracasadas hasta la defensa acorde a fines exitosa.

Desestimación del afecto y desmentida exitosas.

En la segunda tentativa de sintonía por el terapeuta, el paciente había pasado desde las defensas ya mencionadas hasta la defensa acorde a fines exitosa.

Desmentida exitosa.

Desestimación del afecto y desestimación de la realidad y la instancia paterna exitoso-fracasadas.

Desmentida exitosa.

Desmentida exitosa. 


\section{Discusión}

El intento de recabar información y la tentativa de sintonizar con el paciente parecen ser las bases para la orientación clínica subsecuente del terapeuta. La primera intervención central, tendiente a sintonizar con el paciente, enfoca más un estado que un proceso. La investigación de procesos es más frecuente en psicoterapia, y tiene un carácter dinámico: el paciente dice o hace tal cosa guiado por tales conflictos, o debido al uso de tales defensas, etc. El estudio de los estados parece tener un carácter menos preciso: los estados pueden estar sofocados y, para tener acceso a ellos el terapeuta puede tener que apelar a procesos psíquicos sofisticados. Algo similar ocurrió en el terreno lingüístico: la formalización del análisis de las transformaciones (Greimas, 1966) fue previa a una revisión más sofisticada de los estados (Greimas \& Fontanille, 1994).

La sintonía del terapeuta con el paciente involucra, por un lado, un proceso de identificación, que frecuentemente toma en cuenta el estado afectivo (a veces el estado somático) en el momento de la sesión y, por otro lado, un trabajo intelectual para detectar la escena conectada con dicho afecto. La detección de la escena específica (entre las muchas que el paciente suele relatar) requiere de la sensibilidad del terapeuta, que deriva, entre otros factores, del propio análisis y de la posibilidad de procesar las propias defensas patológicas, que muy a menudo son despertadas por las manifestaciones del paciente. La sintonía que el terapeuta intenta establecer en la primera sesión se relaciona con el estado del paciente en ese momento, y parece ser clave para entender otras instancias, no necesariamente pertenecientes a la sesión sino también con escenas extra-transferenciales. De esta manera, el terapeuta puede disponer de una orientación para formular intervenciones referidas a momentos pasados del paciente, así como a escenas actuales de las relaciones extra-transferenciales.

El presente estudio permite inferir que la sintonía involucra seis actividades diferentes que el terapeuta puede realizar: (1) preguntar al paciente qué está sintiendo, (2) preguntar si el paciente siente un afecto específico (que el terapeuta menciona), (3) interpretar un afecto que el paciente no describe, (4) preguntar al paciente por un estado somático, en lugar de la cualidad, del matiz afectivo, (5) preguntar si el paciente experimenta determinado estado somático, y (6) interpretar al paciente un estado somático que el paciente no describe.

En todos los casos estudiados las dificultades surgidas en el intento de sintonizar con el paciente fueron una consecuencia de las defensas patológicas del paciente, desplegadas durante la primera sesión. Como interferencias en la labor clínica, tuvieron mayor eficacia las defensas patológicas que durante la sesión resultaron exitosas o exitoso-fracasadas.

Además es conveniente tomar en cuenta la resistencia del propio terapeuta, así como sus intervenciones clínicamente no pertinentes, que lo hacen correr el riesgo de ocupar el papel de un personaje en alguna escena decepcionante de la vida psíquica del paciente (enactment clínico resistencial).

Como el análisis ha permitido estudiar las defensas del paciente en sus relaciones extra-transferenciales y durante la sesión, fue posible constatar que algunos de los pacientes presentaban un sistema defensivo patológico fracasado en sus relaciones extra-transferenciales (razón por la cual habían consultado), pero que durante la sesión trataron de reinstalar esos mismos mecanismos patológicos en su versión exitosa, con lo cual se incrementaba la resistencia.

También se advierte que las dificultades del terapeuta para sintonizar con el paciente no coinciden tanto con la severidad del diagnóstico y de las defensas, cuanto con el éxito o el fracaso de estas últimas.

Respecto de la categorización de las dificultades en sintonizar con el paciente, es posible diferenciar estas alternativas referidas al terapeuta: 1) su capacidad para rectificarse y 2) la posibilidad o imposibilidad de desarrollar una estrategia clínica pertinente que incluya no solo la sintonía sino también el siguiente paso.

Tomando en cuenta estos comentarios, es posible proponer que la interrupción prematura del tratamiento por parte de Corina, Serafín, Froilán y Norberto, así como la forma insatisfactoria en que terminó la entrevista con Z, llevan a sugerir dos factores que permiten predecir la viabilidad o no del tratamiento: 1) el estado de las defensas del paciente (y sus cambios) durante la sesión, y 2) las dos alternativas recién mencionadas, que corresponden a las capacidades del terapeuta para rectificarse y para desarrollar estrategias clínicas pertinentes para continuar con el trabajo clínico. 


\section{Referencias}

Bohart, A. \& Grenberg, S. (Ed.). (1997). Empathy reconsidered. New directions in psychotherapy. Washington: American Psychological Association.

Bolognini, S. (2004). La empatía psicoanalítica. Buenos Aires: Lumen.

Eagle, M. \& Wolitzky, D. (1997). Empathy: A psychoanalytic perspective. En A. Bohart \& L. Greenberg (Comps.), Empathy reconsidered. New directions in Psychotherapy (pp. 217-244). Washington: American Psychological Association.

Ferenczi, S. (1928). The elasticity of psycho-analytic technique. En S. Ferenczi (Ed.), Final contributions to the problems and methods of psycho-analysis (pp. 87-102). Londres: Hogarth and the Institute of Psycho-Analysis.

Freud, S. (1913). Obras completas. Sobre el inicio del tratamiento. Buenos Aires: Amorrortu Editores.

Freud, S. (1918). Obras completas. De la historia de una neurosis infantil. Buenos Aires: Amorrortu Editores.

Greenson, R. (1965). The working alliance and the transference neurosis. Psychoanalytic Quarterly, 34, 155-181. doi:10.1002/j.2167-4086.2008.tb00334.x

Greimas, A. (1966). Semántica estructural. Madrid: Gredos.

Greimas, A. \& Fontanille, J. (1994). Semiótica de las pasiones. México: Siglo XXI.

Kantrowitz, J., Katz, A., Greenman, D., Morris, $H_{\text {., Pao- }}$ litto, F., Sashin, J. \& Solomon, L. (1989). The patient-analyst match and the outcome of psychoanalysis: A pilot study. Journal of the American Psychoanalytic Association, 37, 893-920. doi:10.1177/000306518903700402

Kohut, H. (1959). Introspection, empathy and psychoanalysis. An examination of the relationship between mode of observation and theory. Journal of the American Psychoanalytic Association, 7, 459-483. doi:10.1177/000306515900700304

Lacan, J. (1957-1958). D'une question préliminaire à tout traitement possible de la psychose. París: Écrits, aux Éditions du Seuil.

Maldavsky, D. (1986). Estructuras narcisistas. Constitución y transformaciones. Buenos Aires: Amorrortu Editores.

Maldavsky, D. (1992). Teoría y clínica de los procesos tóxicos. Buenos Aires: Amorrortu Editores.

Maldavsky, D. (2008). Un análisis de tres niveles (estructura psíquica, intrasesión, intersubjetividad) de una primera sesión de 15 años atrás (Ms. Smithfield) usando el algoritmoDavid Liberman (ADL). Investigaciones en Psicología, 13(2), 79-116. Recuperado de http:// studylib.es/doc/6400226/1-un-an\%C3\%A1lisisde-tres-niveles--estructura-ps\%C3\%ADquica-intra...

Maldavsky, D. (2013). ADL Algoritmo David Liberman, un instrumento para la evaluación de los deseos y las defensas en el discurso. Buenos Aires: Paidós.

Maldavsky, D., Aguirre, A., Alvarez, L., Bodni, O., Britti, A.M., Buceta, C., ... \& Widder, F. (2005). Systematic research on psychoanalytic concepts and clinical practice: the David Liberman algorithm (DLA), Buenos Aires: UCES.

Maldavsky, D., Aguirre, A., Alvarez, L., Bodni, O., Britti, A., Buceta, C., ... \& Varela, R. (2007a). La intersubjetividad en la clínica psicoanalítica. Investigación sistemática con el algoritmo David Liberman ( $A D L)$. Buenos Aires: Editorial Lugar.

Maldavsky, D, Aparain, A., Berenstein, R., Bodni O., Cusien, I., Falise, ... \& Tate de Stanley, C. (2007b). El caso Z (Donnet y Green) revisitado. Investigación sistemática de las erogeneidades y las defensas en el contexto intersubjetivo con el algoritmo David Liberman $(A D L)$. Clínica e investigación relacional. Revista electrónica de psicoterapia, 1, 192-224. Recuperado de http://dspace.uces.edu.ar:8180/ xmlui/bitstream/handle/123456789/1655/Maldavsky_otros_2008_Clinica-1-1.pdf?sequence=1

Maldavsky, D., Cantis J., de Durán, R. \& García Grigera, H. (2007c). Un caso de afasia estudiado con el algoritmo David Liberman (ADL). Acta Psiquiátrica, 54(2), 96-110. Recuperado de http://biblioteca.psi.uba.ar//cgi-bin/koha/opac-detail. pl?biblionumber $=43616$

Maldavsky, D., Cantis J., de Durán, R. \& García Grigera, H. (2007d). Un caso de afasia con fluencia motriz y paraverbal. Summa Psicológica, 5, 101-119. doi:10.18774/summa-vol5.num1-226

Maldavsky D., Cantis J., de Durán R. \& García Grigera H. (2007e). Tres casos de afasia: investigación de las erogeneidades, las defensas y su estado con el algoritmo David Liberman (ADL). Subjetividad y procesos cognitivos, 10, 79-138. Recuperado de http://biblioteca.psi.uba.ar//cgi-bin/koha/opacdetail.pl?biblionumber $=43597$

Maldavsky, D., Argibay, J. C., Scilletta, D., Rembado, J. M., De Simone, L., Otálora, J. \& García Grigera, H. (2016). Estudio de las intervenciones de un mismo terapeuta con 20 pacientes en la primera sesión. Subjetividad y procesos cognitivos, 20, 128-146. Recuperado de http://www.scielo.org.ar/scielo.php?script=sci_ar ttext\&pid=S1852-73102016000100007

McDougall, J. (1978). Primitive communication and the use of countertransference. Contemporary Psychoanalysis, 14, 173-209. doi:10.1080/00107530.1978.107455 34 
Olden, C. (1958). Notes on the development of empathy. The Psychoanalytic Study of the Child, 13, 505-518. Recuperado de http://www.tandfonline.com/doi/ab s/10.1080/00797308.1958.11823193?journalCode= upsc20

Pigman, G. (1995). Freud and the history of empathy. The International Journal Psycho-Analysis, 76, 237-254. Recuperado de https://www.ncbi.nlm.nih.gov/ pubmed/7628894

Schafer, R. (1959). Generative empathy in the treatment situation, The Psychoanalytic Quarterly, 28, 342373. Recuperado de http://psycnet.apa.org/record/1960-04354-001

Trop, J. \& Stolorow, R. (1007). Therapeutic empathy: An intersubjective perspective. En A. Bohart \& L. Greenberg (Eds.), Empathy reconsidered. New directions in psychotherapy (279-291). Washington: American Psychological Association.

Widlöcher, D. (2004). Dissection de l'empathie, Revue Française de Psychanalyse, 48(3), 981-991. Recuperado de https://www.cairn.info/revue-francaise-depsychanalyse-2004-3-page-981.htm 